\title{
Super-Resolution PIV by Recursive Local-Correlation
}

\author{
Douglas P. Hart \\ Massachusetts Institute of Technology \\ Department of Mechanical Engineering, Room 3-246 \\ Cambridge, MA 02139-4307 \\ E-mail: dphart@mit.edu
}

\begin{abstract}
This paper presents a method of accurately determining the displacement of tracer particles between two images using recursive correlation. The local correlation value is iteratively arrived at through successive approximations of local displacement using increasingly smaller regions of determination. By starting with a large search area and iteratively narrowing the search restricting the search after each iteration based on the resulting calculation, very high-resolution PIV processing can be achieved. Spurious vectors are eliminated and accuracy and processing speed is maintained by correlating images in compressed (sparse array) format using second-order spatial correlation. The methodology of this unique image analysis method is presented along with a discussion of its limitations and applicability.
\end{abstract}

Keywords: Super-Resolution, PIV, Correlation

\section{NOMENCLATURE}

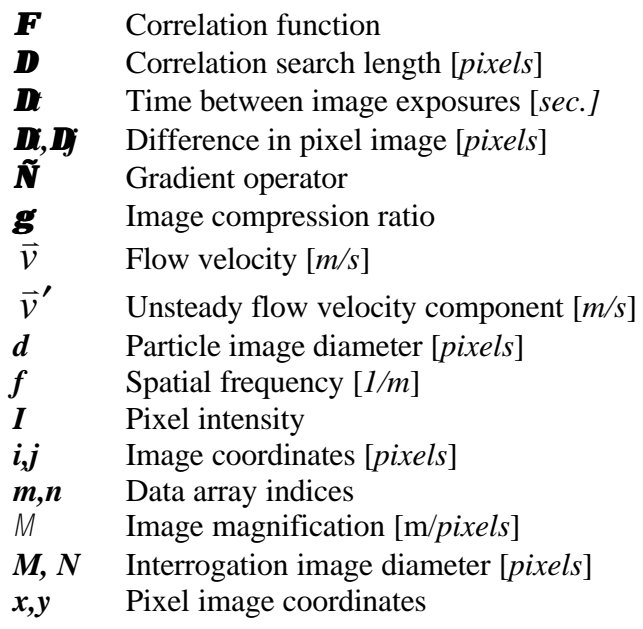

\section{INTRODUCTION}

The probability of obtaining a statistically meaningful measure of local flow velocity using correlation increases as the number of tracer particles and pixels used to image tracer particles increases. Correlations based on small data sets are unlikely to yield meaningful result in all but ideal circumstances. If a large data set is used for an individual correlation, 
however, spatial resolution is severely limited and the ability to resolve large regions of flow is restricted by the resolution of current imaging technology. To overcome this, investigators are forced to correlate relatively small data sets and rely on image characteristics to insure a high probability of uniqueness for the resulting correlation value. This limitation can be circumvented by using a recursive correlation algorithm. That is, the local displacement can be approximated by correlating a fairly large data set before reducing the data set and recorrelating the region limiting the search for the maximum correlation value based on the previous correlation. Unfortunately, any error in approximating the local-correlation value in this manner propagates resulting in unacceptable errors in the results. Furthermore, the multiple correlations of subregions required to obtain any single vector greatly slows processing.

\section{Background}

Particle image velocimetry is inherently limited by the resolution of current imaging technology and the statistical nature by which PIV images are processed. Specifically, it is limited by the nature of the determination of tracer particle movement by local-correlation. Westerweel, in a recent publication [1998], demonstrates that the minimum pixel number required to describe a single tracer particle is $2 p x \times 2 p x$. Fewer than this and the ability to resolve sub-pixel displacement begins to breakdown. Such a limitation, however, has more to do with the nature of current imaging technology than it does with an inherent physical limitation of PIV. As optical diffraction limits the minimum size of the projected image of a tracer particle, it follows that the limit to which any giving discreet imaging system can resolve a volume of flow is limited by the size and spacing of the pixel sensors rather than the total resolution of the array. There is, however, a direct relationship between sensor area and the sensitivity to light. Consequently, there exist a practical limit to the volume that can be imaged. Interestingly, this limit has little to do with the absolute spatial-resolution with which the flow within this volume can be resolved. That is, it is the diffraction size of the optics that limits the spatial-resolution and not the total number of pixels in the image array.

Optical diffraction limitations are inherent to all particle tracking velocimetry techniques and not just PIV. However, PIV by the statistical way in which particle displacements are determined introduces another, somewhat artificial limitation. As Keenan and Adrian [1987] demonstrated, PIV requires roughly ten tracer particle images per interrogation region to accurately resolve local particle displacement using traditional correlation. This limitation is artificial in that a single pair of particle images contains enough information to resolve the local flow velocity. It is only that the displacement of a single pair of particle images can not be resolved through correlation except in the most ideal of circumstances, when only one particle image exists within an interrogation region, that limits spatial resolution. When a flow is heavily seeded, however, it becomes impossible to match pairs of particle images. Consequently, when attempting to optimize accuracy and spatial-resolution, a careful balance exists. If the seeding density is low, particle tracking technology can be used to follow individual particles but the seeding density itself severely limits spatial resolution as few particles exist within the interrogation region. At high seeding densities, correlation is required to resolve displacement and thus, spatial-resolution is limited by the number of particle image pairs needed to insure a high probability of uniqueness of the solution. This limitation can be overcome by coupling particle-tracking technology with PIV localcorrelation processing as first presented by Keenan and Adrian [1995]. Such a coupling allows relatively high seeding densities, higher than can be tolerated by particle tracking alone while maintaining the accuracy of particle tracking. This type of analyses, however, 
fails to resolve images to the limits of available information. Although an improvement, the nature of such a coupling is still limited by the same conditions that limit particle tracking on a larger scale. It, however, circumvents the first order difficulties of high seeding densities at the course scales typical of PIV processing. At extremely high seeding densities, particle overlap, agglomeration, diffraction, and distortion of light, prevent the use of particle tracking even at the smallest scales. It becomes impossible to resolve any single particle image in the flow. Yet, it is at these seeding densities where the greatest possible information content exists within an imaged flow, a condition where the addition of more particles to a flow reduces the probability of obtaining a valid correlation from a finite region rather than increases it. Ideally, any super-resolution processing algorithm should be able to handle both, very heavily seeded flows as well as very sparse flows with equal robustness and speed. This paper presents such an algorithm, a super-resolution algorithm based entirely on localcorrelation.

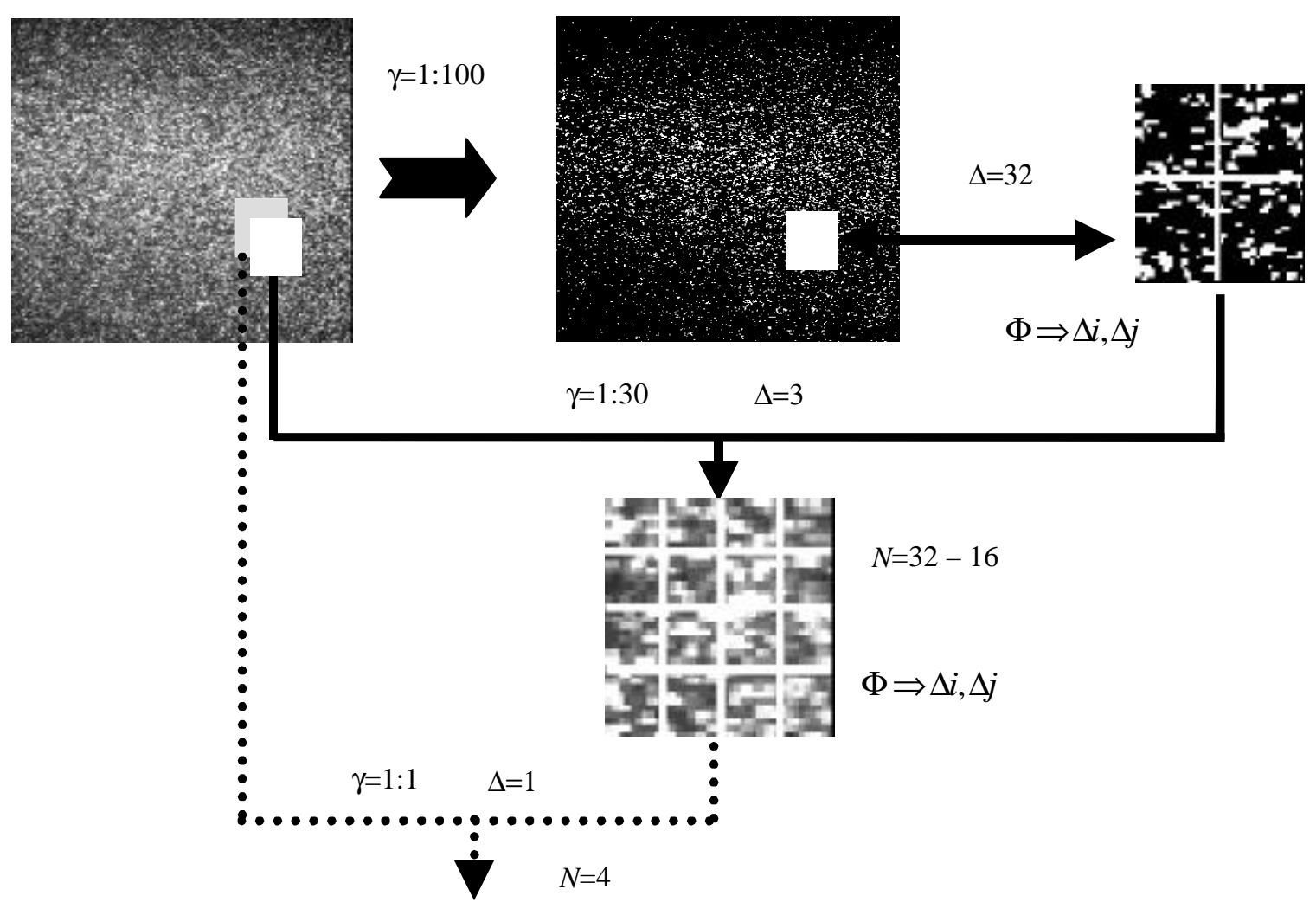

Figure 1: Super-Resolution processing through recursive correlation is carried out by correlating a standard size region then splitting the region not smaller subregions before re-correlating using the initial correlation as a predictor of the subsequent correlation. This recursive correlation process can be carried out down to the individual particle size.

\section{METHODOLOGY}

Recursive correlation is, in principle, relatively simple. Correlation is carried out in the same way traditional correlation processing is carried out. That is, a subregion is selected, the region is correlated, and the peak correlation is determined. Unlike traditional correlation, the resulting value is stored and the subregion is broken into smaller regions that are, in turn, correlated to yield the velocity over a reduced region of determination. The previous 
correlation is used as an estimate to limit the search for the current correlation (see Fig. 1). In this way, the probability of locking onto the correct displacement is maintained at a high level despite the small data sets. This iterative process can be carried out down to the dimensions of individual particle images.

The inherent difficulty with recursive correlation and the reason it has not been widely used is that all errors propagate down to the smallest scale. That is, if an error in the initial correlation occurs, this error limits the search for subsequent correlations to regions in which the correct particle displacement may not exist. Consequently, the peak correlation found in subsequent correlations may not represent the actual displacement of the particles in the image. Furthermore, any single vector can be the result of ten or more individual correlations. This can slow processing to a level where the technique becomes impractical. In order for recursive correlation to be viable, a very fast highly robust processing algorithm is needed.

\section{RESOLUTION ENHANCEMENT}

The spatial resolution that can be obtained from PIV images is inherently limited by the statistical nature of correlation based processing. In dividing images into subregions, a careful balance must be maintained between the statistical number of tracer particle images within the subregions and the relative displacement of particle images between exposures from one area of a region to another. If a subregion is too small, too few tracer particle images exist within the region and a statistically meaningful result for averaged flow velocity can not be obtained through correlation. If the subregion is too large, however, the displacement of tracer particles between exposures from one area of the region to another due to local flow gradients can be greater than the image diameter of the tracer particles. This condition results in a decrease in signal-to-noise ratio due to displacements in individual particle correlation peaks too large to add to the net correlation signal [Keane, Adrian, 1992; Hart, 1996]. Consequently, there exists an optimum correlation window size that depends on the local flow conditions and seeding density. The optimum window size is rarely constant from one region of a flow to another. Thus, traditional PIV processing in which the interrogation window size is fixed, results in the majority of processing being carried out with less than optimum interrogation windows.

The highest spatial resolution that can be obtained by tracking tracer particles in a flow is of the order of the diameter of a single tracer particle image. To achieve this level of spatial resolution through correlation, interrogation windows with a size equal to the tracer particle image diameter must be used. Such window sizes can not generate statistically meaningful results unless it is known a priori that the local displacement is within a few pixels. A correlation based on such data results in a peak value representing the optimum match of the shape of individual particle intensity profiles rather than a statistical spatial match of distinct randomly space points. This is the bases for super-resolution PIV processing through localcorrelation. The interrogation size and the search distance are iteratively reduced until the smallest meaningful scale is reached. Each iteration is based on the results of the previous calculation. Thus, a priori information exists enabling statistically meaningful results despite inordinately small interrogation window sizes.

There is, however, a limit to the spatial resolution that can be achieved by recursive correlation that does not necessarily represent the seeding density of the flow nor the characteristics of the way in which the flow was imaged. This limit is the result of the maximum velocity gradient that can exist to resolve local tracer particle displacement from a 
finite interrogation region through correlation. If an initial correlation window size is selected so that there exists a statistically meaningful number of tracer particle images, roughly ten (see Keane and Adrian [1992]), then there exists a corresponding maximum local velocity gradient that can be resolved. This limit in velocity gradient is approximately $\left|\nabla \vec{v}_{i j}\right|_{\max } \cong \frac{d}{N \Delta t}$ or a total displacement from one area of an interrogation region to another of roughly one particle image diameter [Hart, 1998]. Because each iteration of recursive correlation processing relies on the outcome of the previous correlation, the maximum velocity gradient that can be resolved depends on the average velocity gradient of the previous solution. Consequently, if a flow is sparsely seeded such that the initial correlation window size must be relatively large to contain ten tracer particle images, improvement in spatial resolution through recursive correlation may be futile. Each subsequent correlation increases the spatial frequency of the velocity that can be resolved but does not effect the ability to resolve the magnitude of the unsteady velocity component at lower frequencies. The maximum unsteady velocity component that can be resolved depends on the spatial frequency, $f$, of the velocity component and the size of the correlation window used to resolve it; $\left|\vec{v}_{i j}^{\prime}\right|_{\max }=\frac{d}{\Delta t n f 2 \pi}$ where $f \leq \frac{1}{2 n \mathrm{~m}}$ and $\left|\vec{v}_{i j}^{\prime}\right|$ is the unsteady velocity component in the $i j$ interrogation plane such that $\vec{v}_{i j}=\overline{\vec{v}}_{i j}+\vec{v}_{i j}^{\prime}$ and $n$ here defines the interrogation window size. Because the unsteady velocity component at low spatial frequencies is limited by the largest interrogation size which is equal to the initial correlation window size, $N$, the magnitude of the unsteady velocity component that can be resolved by recursive correlation at all spatial frequencies is

$$
\left|\vec{v}_{i j}^{\prime}\right|_{\max }=\frac{\mathrm{m} d}{\Delta t \pi}
$$

This is inversely proportional to the accuracy with which particle displacement can be determined. Consequently, a tradeoff must be made between accuracy, spatial resolution, and the ability to resolve unsteady velocity.

\section{COMPUTATIONAL INTENSITY}

Consider the direct correlation function defined by the equation;

$$
\Phi_{\Delta i, \Delta j}=\frac{\sum_{m=1}^{N} \sum_{n=1}^{N}\left[I_{m+\Delta i, n+\Delta j} \cdot I_{m, n}\right]}{\sqrt{\sum_{m=1}^{N} \sum_{n=1}^{N} I_{m, n}^{2}} \cdot \sqrt{\sum_{m=1}^{N} \sum_{n=1}^{N} I_{m+\Delta i, n+\Delta j}^{2}}}
$$

The computational cost of processing this function can be defined by the number of individual pixel comparisons plus the number of array interrogations needed to determine the peak correlation from the resulting correlation table. Thus, the computational cost of processing an $N x N$ interrogation region is $4\left(N^{2} \Delta^{2}+\Delta^{2}\right)$ where $\Delta$ is the magnitude of the distance searched for the peak correlation in terms of pixels (A $\pm 32 p x$ search implies $\Delta=32$ ). The computational cost of using the direct correlation function for super-resolution PIV through recursive correlation is thus equal to $4 N^{2} \Delta^{2}+4\left(\frac{N}{2}\right)^{2} \alpha^{2}+4\left(\frac{N}{4}\right)^{2}\left(\frac{\alpha}{2}\right)^{2}+4\left(\frac{N}{8}\right)^{2}\left(\frac{\alpha}{4}\right)^{2}+\ldots$ or $4 N^{2} \Delta^{2}+N^{2} \alpha^{2}\left[1+\frac{1}{16}+\frac{1}{256} \ldots\left(\frac{2}{N}\right)^{4}\right]$ where $\alpha$ is the maximum pixel displacement gradient that can 
be tolerated in the original correlation $(\alpha \cong d)$. As $N$ becomes large, this approximately equals $4 N^{2}\left(\Delta^{2}+\frac{4}{15} \alpha^{2}\right)$. The computational intensity of the search for the maximum correlation value within the correlation table after each correlation is $4 \Delta^{2}+4 \alpha^{2}\left[1+\frac{1}{4}+\frac{1}{16} \cdots\left(\frac{2}{N}\right)^{2}\right]$. This approaches $4\left(\Delta^{2}+\frac{1}{3} \alpha^{2}\right)$ as $N$ increases. Thus, the net computational intensity for large values of $N$ is roughly $4 N^{2}\left(\Delta^{2}+\frac{4}{15} \alpha^{2}\right)$ or $\frac{4}{15}\left(\frac{\alpha}{\Delta}\right)^{2}$ times that of the initial subwindow correlation. Because $\alpha$ is typically much less than $\Delta$, the computational difference between superresolution processing and standard processing is negligible.

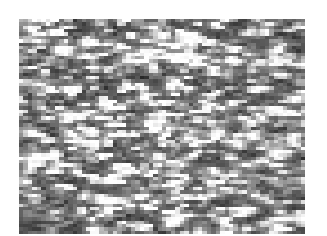

$\gamma=1: 1$

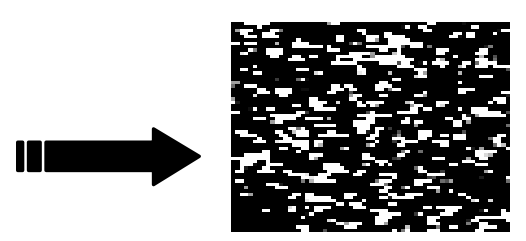

$\gamma=1: 100$
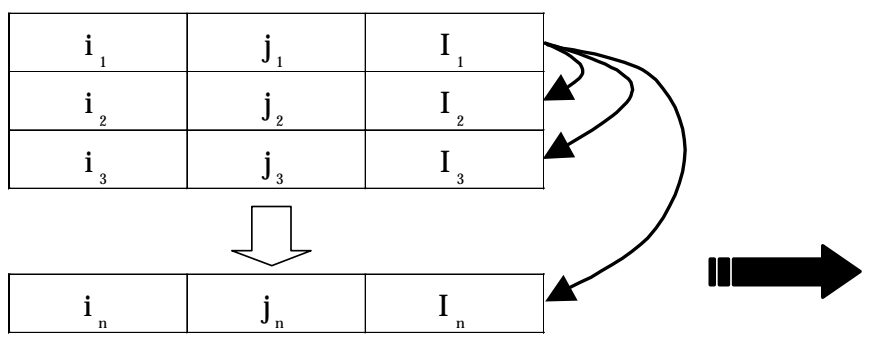

$\Delta$

$$
\begin{aligned}
& \text { FFT: } \sim 3 \mathrm{~N}^{2} \log (\mathrm{N})+\mathrm{N}^{2} \\
& \text { Sparse: } \sim(\gamma \Delta \mathrm{N})^{2} \\
& (\gamma \Delta)^{2}<<1
\end{aligned}
$$

$\gamma \equiv$ compression ratio

$\Delta \equiv$ search distance

$\mathrm{N} \equiv$ correlation window size

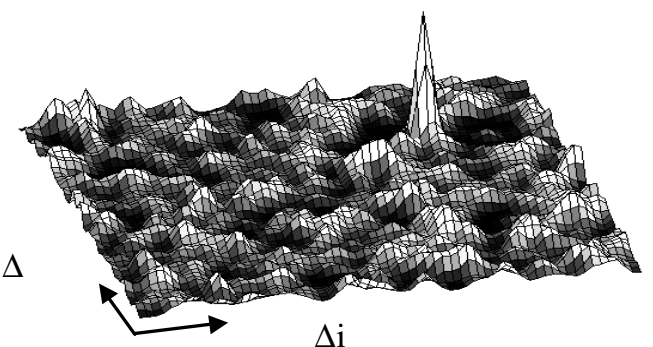

Figure 2: Compression based correlation. The images are compressed and each pixel's intensity is stored into a sparse array. The images are then correlated by comparing individual entries within the array(s). Correlation rates far greater than FFT based correlation can be realized with this algorithm.

If a compression based processing algorithm is utilized [Hart, 1998] where the total number of pixels in the data set is reduced by a factor $\gamma$ before correlation, super-resolution processing can be carried out far faster than it takes to process the same image set using an FFT based algorithm at normal resolution (see Fig. 2). Since the additional resolution is gained at little computation cost, the compression ratio can be reduced between each iteration such that there is no compression during the final correlations and thus, no data loss. Consider the case in which the number of pixels after compression is maintained from one iteration to the next such that the compression ratio is dropped by a factor of four after each iteration. The net computational cost then equals the correlation computational cost, $4 \gamma^{2} N^{2} \Delta^{2}+4 N^{2} \gamma^{2} \alpha^{2}\left[1+\frac{1}{4}+\frac{1}{16} \cdots\left(\frac{2}{N}\right)^{2}\right]$ where $\gamma$ is the compression factor plus the computational cost associated with the search for the peak correlation. Thus, the net computational cost for super-resolution cross-correlation processing using sparse array image correlation is 


$$
4 \Delta^{2}\left[1+\frac{4}{3}\left(\frac{\alpha}{\Delta}\right)^{2}\right]\left(1+\gamma^{2} N^{2}\right)
$$

For typical values of $\alpha / \Delta, 4 \Delta^{2}\left[1+\frac{4}{3}\left(\frac{\alpha}{\Delta}\right)^{2}\right]\left(1+\gamma^{2} N^{2}\right) \cong 4 \Delta^{2}\left(1+\gamma^{2} N^{2}\right)$ which is the computational cost associated with sparse array correlation of non-recursively processed images. As an example, the computational cost for a correlation with $\mathrm{N}=32 p x, \Delta=16 p x, \alpha=8 p x$, and an initial compression ratio of 50:1, is roughly one-fifth that of standard FFT processing even when correlated down to single particle levels $(4 x 4 p x)$. This is an increase in spatial resolution of about a hundred. Thus, super-resolution processing can be carried out at rates higher than FFT correlation without sacrificing accuracy due to image compression.

\section{ERROR CORRECTION}

Currently, the most widely used and accepted technique to eliminate correlation errors is to compare vectors with their neighbors to determine if they are in some statistical or physical sense inconsistent. This technique, analyzed in detail by Westerweel (1994), is based on the assumption that vectors resulting from correlation errors are far removed in magnitude and/or direction from neighboring vectors. It assumes that the resolution of PIV data is high enough and the flow features benign enough that apparent discontinuities in the flow will not present themselves and be eliminated. It is a method of detecting errors and not a method of resolving tracer particle displacement. Detailed correlation information is discarded before interrogation. Consequently, errors can only be eliminated from the results and replaced by interpolated values. Furthermore, this error correction method addresses only the most obvious of correlation errors and does not address the more subtle problems that severely limit sub-pixel accuracy and resolution. Although extremely useful, post-interrogation error correction is not ideal.

Both errors resulting from insufficient data and errors caused by correlation anomalies can be eliminated during processing, regardless of the method of correlation, simply by multiplying the correlation table generated during processing by the correlation table generated from one or more adjacent regions;

$$
\Phi_{\Delta i, \Delta j}^{\prime}=\frac{\sum_{m=1}^{N} \sum_{n=1}^{N}\left[I_{m+\Delta i, n+\Delta j} \cdot I_{m, n}\right]}{\sqrt{\sum_{m=1}^{N} \sum_{n=1}^{N} I_{m, n}^{2}} \cdot \sqrt{\sum_{m=1}^{N} \sum_{n=1}^{N} I_{m+\Delta i, n+\Delta j}^{2}}} \cdot \frac{\sum_{o=1}^{N} \sum_{p=1}^{N}\left[I_{o+\Delta i, p+\Delta j} \cdot I_{o, p}\right]}{\sqrt{\sum_{o=1}^{N} \sum_{p=1}^{N} I_{o, p}^{2}} \cdot \sqrt{\sum_{o=1}^{N} \sum_{p=1}^{N} I_{o+\Delta i, p+\Delta j}^{2}}}
$$

This correlation error correction technique is illustrated in Fig.3. Here, the correlation table calculated during processing of one region (Fig. $3 a$ ) is multiplied, element-by-element, by the correlation table calculated from an adjacent region that overlaps the first region by fiftypercent (Fig. 3b). Neither of the correlation tables in this example (Fig. 3a or 3b) has a discernable peak representing tracer particle displacement. The resulting correlation table (Fig. $3 c$ ), however, has very few correlation anomalies and has a very prominent correlation peak in the lower right hand corner. 


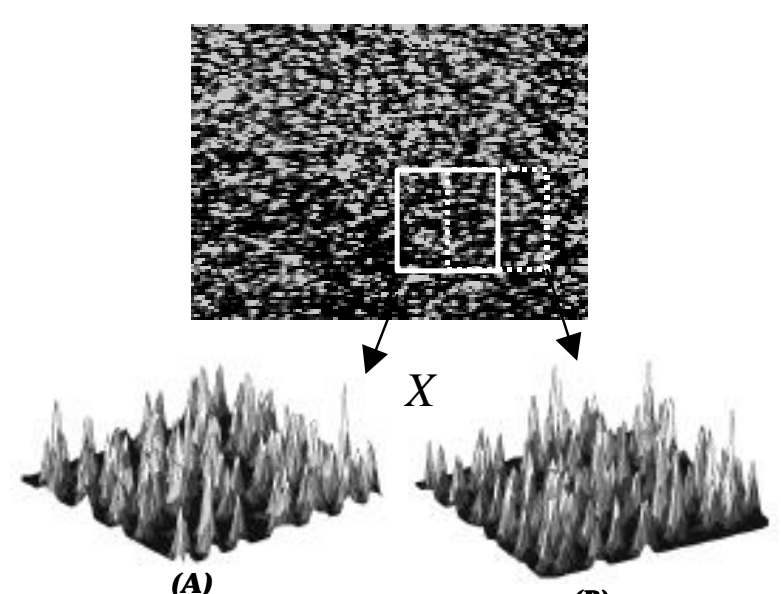

(B)

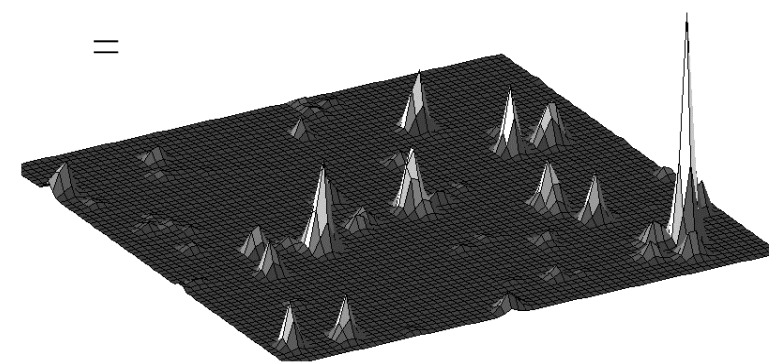

(C)

Figure 3: Elimination of correlation anomalies by multiplying the correlation tables from adjacent regions. Correlation values that do not appear in both tables are eliminated allowing tracer particle displacement to be resolved.

Correlation error correction is effectively a correlation of two or more correlation tables. It is not an averaging technique. Any correlation value that does not appear in the combined correlation tables is eliminated from the resulting table. As the probability of exactly the same anomalies appearing in different regions is very small, correlation anomalies, regardless of their source, are eliminated from the data. Conversely, correlation values that are identical in location and magnitude in the combined tables are amplified. Thus, even if tracer particle displacement is not discernable in any of the combined correlation tables, multiplied together, the peak is either easily resolved or it becomes evident that at least one of the combined tables does not contain sufficient information to resolve particle displacement.

Correlation error correction is not equivalent to correlating a larger region equal to the sum of the combined regions. Such a correlation would not eliminate correlation anomalies. It would, assuming no local velocity gradient, only strengthen the correlation peak representing the average particle displacement in the combined regions. This is not true of correlation error correction. The correlation peak found in the table resulting from correlation error correction is weighted to the displacement of the tracer particles within the overlap of the combined regions. Information within the overlapping regions identically effect the values in all of the correlation tables equally and are, therefore, not removed during processing. Particle displacements in regions outside the overlap influence the calculated displacement but to an extent that depends on the similarity in displacement. Thus, rather than a reduction in resolution, there is an improvement that depends on the size of the overlap and the gradient of the velocity relative to the size of the sample volume. 
When second-order correlation is combined with recursive super-resolution processing, significant improvements in spatial resolution can be realized as illustrated in the example shown in Fig. 4.

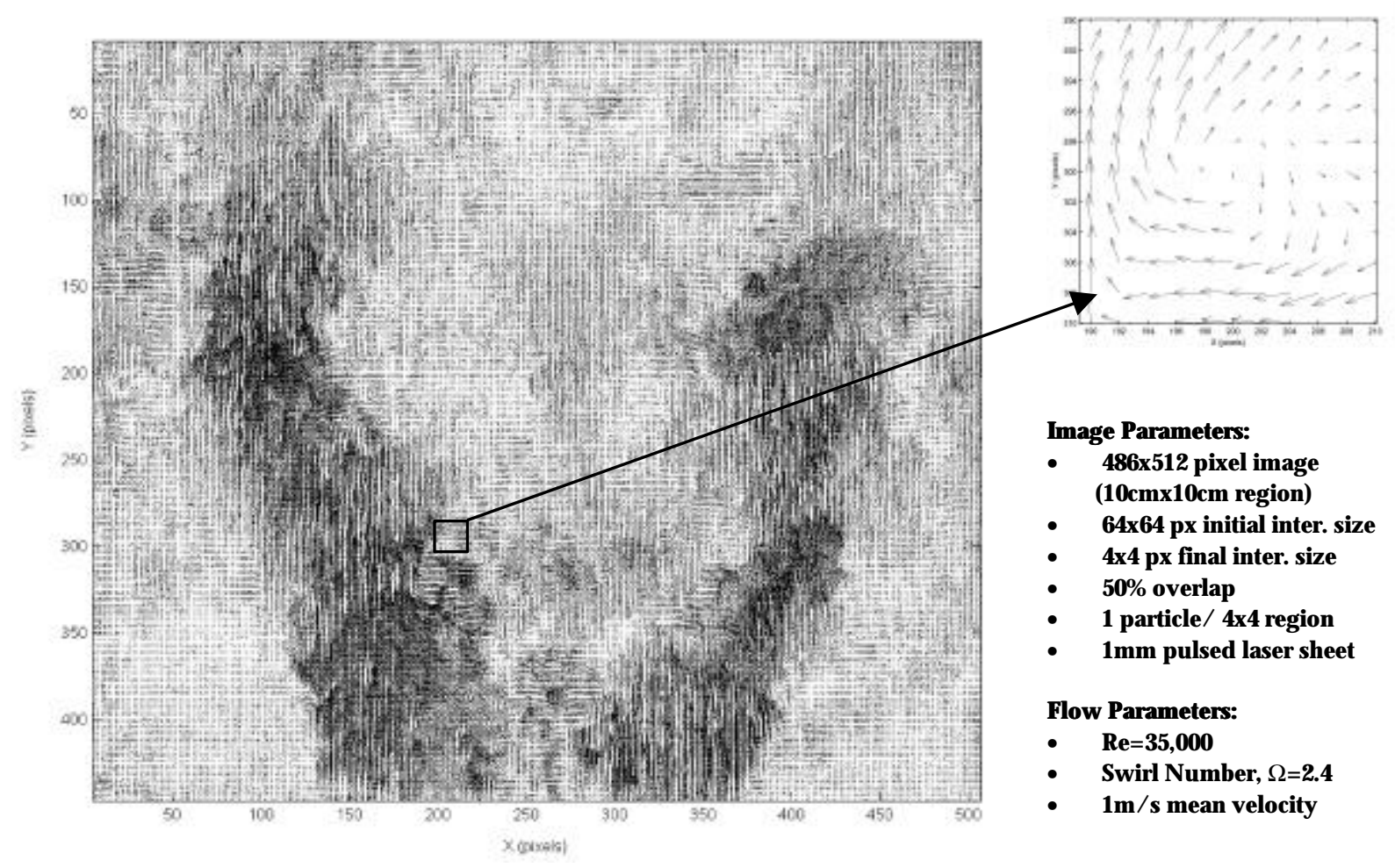

Figure 4: 60,000 vectors calculated using recursive correlation from a $486 \times 512$ image of a swirling flow ( $R e=35,000, \Omega=2.4)$ undergoing sudden expansion. Each vector on average represents a single tracer particle in the flow and is the result of as many as five sub-window correlations. These results are processed at a rate of over 1,000 vectors/sec using sparse array image correlation with correlation error correction.

\section{CONCLUSIONS}

The spatial resolution of particle image velocimetry, PIV, can be significantly improved through recursive correlation. By breaking PIV images into subregions, correlating each subregion before reducing the size of the subwindows and re-correlating using the results of the previous correlation to narrow the search for subsequent correlations, improvements in spatial resolution by factors greater than a hundred can be realized. The computational cost of such processing is minimal. Through the use of compression based correlation, superresolution PIV processing can be done faster than spectral, FFT, based processing at typical resolutions. Furthermore, because the computational costs associated with compression based correlation are greatly dependent on the number of pixels being correlated, the majority of computation is done during the initial subregion correlation. Subsequent correlations can be performed at much reduced compression ratios improving subpixel accuracy without sacrificing computational speed. Traditionally, recursive algorithms of this type are subject to sever difficulties as any error that occurs in determining particle displacement can then be 
propagated to the smallest scale. Through a unique second-order correlation algorithm, such errors are adverted and the resulting processed data is nearly free of spurious results.

The advantage that a purely correlation based super-resolution algorithm has over hybrid correlation/particle tracking algorithms is that they do not require that individual particles be distinguishable. Consequently, correlation based super-resolution PIV processing can be performed on very heavily seeded images resolving local velocities at spatial resolutions nearing the dimensions of individual particles.

\section{REFERENCES}

Adrian, R. J. (1991) "Particle imaging techniques for experimental fluid mechanics," Annual Review of Fluid Mechanics Vol. 23, pp. 261-304.

Fincham, A. M.; Spedding, G. R. (1997) "Low Cost, High Resolution DPIV for Measurement of Turbulent Flow," Experiments in Fluids., Vol. 23, pp. 449-462.

Fincham, A. M.; Spedding, G. R.; Blackwelder, R. F. (1991) "Current Constraints of Digital Particle Tracking Techniques in Fluid Flows," Bull Am Phys Soc. Vol. 36, 2692.

Hart, D.P., (1998) “High-Speed PIV Analysis Using Compressed Image Correlation,” Journal of Fluids Engineering.

Hart, D.P., (1998) "The Elimination of Correlation Errors in PIV Processing," 9th International Symposium on Applications of Laser Techniques to Fluid Mechanics, July 13-16, 1998, Lisbon, Portugal.

Hart, D.P., (1996) "Sparse Array Image Correlation," $8^{\text {th }}$ International Symposium on Applications of Laser Techniques to Fluid Mechanics, Lisbon, Portugal.

Keane, R. D.; Adrian, R. J.; Zhang, Y (1995) “Super-resolution particle image velocimetry” Measurement Science and Technology, Vol. 2, pp. 1202-1215.

Keane R.D.; Adrian, R.J., (1992) "Theory of cross-correlation of PIV images," Applied Scientific Research, Vol. 49, pp. 191-215.

Keane, R. D.; Adrian, R. J., (1990) “Optimization of Particle Image Velocimeters," Measurement Science and Technology, Vol. 6, pp. 754-.

Landreth, C. C.; Adrian, R. J., (1990) "Measurement and Refinement of Velocity Data Using High Image Density Analysis in Particle Image Velocimetry," Applications of Laser Anemometry to Fluid Mechanics, Springer-Verlag, Berlin, pp. 484-497.

Okamoto, K; Hassan, Y. A.; and Schmidl, W. D., (1995) "New Tracking Algorithm for Particle Image Velocimetry," Experiments in Fluids., Vol. 19, pp. 342-347.

Prasad, A.K.; Adrian, R.J.; Landreth, C.C.; Offutt, P.W., (1992) "Effect of Resolution on the Speed and Accuracy of Particle Image Velocimetry Interrogation," Experiments in Fluids, Vol. 13, pp. 105-116.

Raffel, M.; Kompenhans, J., (1994) "Error Analysis for PIV recording Utilizing Image Shifting," Proc. $7^{\text {th }}$ International Symposium on Applications of Laser Techniques to Fluid Mechanics, Lisbon, July, p. 35.5.

Westerweel, J. (1994) "Efficient Detection of Spurious Vectors in Particle Image Velocimetry Data," Experiments in Fluids., Vol. 16, pp. 236-247.

Westerweel, J.; Dabiri, D.; Gharib, M., (1997) "The Effect of a Discrete Window Offset on the Accuracy of Cross-Correlation Analysis of Digital PIV Recordings," Experiments in Fluids, Vol. 23, pp. 20-28.

Willert, C. E.; Gharib, M. (1991) "Digital Particle Image Velocimetry,” Experiments in Fluids, Vol. 10, pp. 181193. 